mywelf included, have arrived; but, more recently, Professor Boström, of Giessen, reports 1 that he has succoeded in making artificial cultiva. tions on solidified blood-serum, Agar-Agar, and gelatine, that the growths come to maturity in five or six days, and present the typical appesrance of actinomyces. This typical appearance he considers to bo that which has been described as occurring in man, for he believes that the club-shaped elements occur only where the nutritive material is becoming exhausted, or where involntion is taking place. If these observations be confirmed, the organism will have to be classed amongst the schyzomycetes or bacteria, instead of, as heretofore, amongst the hyphomycetes or moulds; this view is taken by Dr. Israel, and is supported by my own specimens.

It has been doubted whether the "rosettes" in cattle are organic at all, whether they are not crystals of fat or calcareous masses formed in caseous areas of inflammation. In the cases observed by myself, the evidence against these views is very strong, since neither acetic or nitric acids, ether, or alcohol, have any destructive effect upon the threads; although, in the specimen given me by Dr. J. Israel, alcohol and ether caused the club-shaped bodies to shrivel, but did not entirely dissolve them ; and, in the second place, heat does not liquefy, or osmic acid stain them; there is, too, the positive evidence that all forms, from the single rod-shaped eloments up to the complete circle of threads with a hollow centre, are to be found often in a single specimen, so that it is not any great assumption to suppose that the larger forms have developed from the smaller ones, not by a process of crystallisation, but of actual growth.

Little is known as to the origin of the disease. Dr. James Israel believes that it may develop from some form of leptothrix found in the mouth; and in the last case which he has had under his care, a hollow tooth was found embedded in the lung under the pleura, and apparently the starting. point of the disease. Jolne has recorded cases in which the disease attacked the spermatic cords of horses which had recently undergone castration : so that there can be little doubt that the organism may be derived from many different sources, and that, when it finds a suitable nidus, it dovelops, and by its growth sets up those processes of inflammation which constitute the disease known as actinomycosis.

\section{ON THE TENDON-REACTIONS.}

BY A. DE WATTEVILLE, M.A., M.D., B.Sc.,

Physician in charge of the Electrical Department, St. Mary's Hospital.

Is the first of Dr. Sharkey's admirable Gulstonian Lectures, there occurs a passage (see British Midedical Journax, March 20th, pp. 532 and 533) of which I gladly avail myself to enforce the views of those who, like myself, ohject to the name of "tendon-reflexes," or "deep reflexes," generally given by medical writers to phenomena more correctly described as "tendon-reactions," or " myotatic contractions." It is true that, as the lecturer observes, the short latency of these muscular spasms, as compared to that of true reflex contractions, is, for those accustomed to physiological methods of investigation, an argument of considerable weight. Many clinical observers, however do not seem to appreciate this kind of proof, and experience no diffi. culty in assuming that certain nervous impulses may, without obvious reason, travel three times as quickly as others along paths similar in every respect, and identical up to a certain point. But the data of measurcment are not the only considerations which make the reflex nature of tendon reactions doubtful ; and my object is to statc, as briefly as possible, the other aspects of the case.

1. I have adverted elsewhere to the fact that true spinal reflexes usually consist in movements of a more or less co-ordinated nature involving more muscles than one. The resson of this fact is, that the motor physiological units in each spinal segment are not representative of single muscles 80 much as of certain actions resulting from muscular combinstions. In that form of contraction which follows an extensile vibration communicated to a tendon, the response is limited to the muscle of which the tendon is submitted to the exciting process, and bears a close analogy to the effect of an electrie or other direct excitation of the muscle. Rtflex actions differ from such manifestations of peripheral irritability, inasmuch as they either poesess a "purposive" charncter, or appear to be survivals of protective movements developed in the course of the evolution of ances. tral forms.

1 Juhresbericht ïber Path. Org., Baumgarten, 18\&6. P. 149.
2. True reflexes occurring in the sphere of voluntary muscle are amenable to volitional inhibition; tendon reactions can be only neutralised by contraction of the antagonists. Hence, our opponents must assume that there are, not only two rates of travelling of nervous impulses, but two different kinds of discharge from the spinal motor cells : one that can be inhibited, the other that can not.

3. The myographic curve of a muscle, excited by extensile vibration, appears to differ somewhat from that of a muscle responding to a sensory excitation, inasmuch as its ascent appears to be more abrupt. (See Butrish Miedical Jovrnal, Nay 20th, 1882.)

4. The diffusion of true spinal reflex processes in the cord differs by obviously different characters from the apparent diffusion of "mya tatic" contractions, which can be shown to depend upon vibrations, transmitted through the bony structures to hypertonic muscles at. tached to them. This point leads me to consider

5. The phenomenon known as the crossed knco-jerk. It is not rarely observed, in cases where the jerk is abnormally marked, that the two legs respond to percussion of one patellar tendon. This has often been triumphantly addnced by our opponents as proof of tho reflex nature of tendon-reactions; but, as I have shown in the paper just quoted, the closer study of this phenomenon furnishes us with most convincing arguments. First, it is to be observed that, on the one hand, contraction of the muscles in the opposite leg, does not occur after percussion of the patellar tendon, unless the position of the experimentee is such as to allow the impulse to bo transmitted through the femur to the pelvis ; that on the other, percussion of the heel also excitcs this contraction when the whole leg is kept extended at a right angle to the pclvis. Flexion of the limb (or fracture of the bone, as Waller has shown in rabbits) arrests this mechanical impulse and, along with it, the crossed muscular contraction. Secondly, it must be noted that the muscles excited in the opposite leg are not the extensors, but the adductors, which, by their anato. mical relations, are those most liable to be stretched by an impact communicated to the pelvis. Thirdly, as I have shown, the direct and crossed contractions occur at the same instant after the percus. sion of the tendon. Now, if there is one fact certain in the range of spinal physiology, it is that a relatively considerable time is required for the transmission of an excitation from one side of the spinal cord to the other; and that a true crossed reflex has a much longer latencs than a direct response. Hence, we have two good reasons for rejecting the reflex nature of the crossed reaction.

Such is, expressed in as few words as possible, the cumulative cvidence upon which we base our vier. that the usual muscular con. tractions obtained by very sudden extension or percussion of tendons are not true reflexes. It is, of course, perfectly possible that, in certain cases of spinal over-excitability, the usual methods employed to elicit them may give rise to true reflected spasms ; but it must not bs forgotten that, in hypertonic conditions of the muscular system, any vibrations, even very slight, imparted to the bony frame-work, may give rise to symptoms simulating the effects of intrasinal diffusion.

Before concluding, I must allude to a defective argument in Dr. Sharkey's discussion of this point. He compares the latency of the closure of the eye to light excitations with that of the knee-jerk ; and, from the similarity of the two, appears to conclude that measurements are, after all, not opposed to the reflex theory of tendon-reactions, But he does not allude to the enormous difference between the length of the nervous paths along which the nervous impulses have to run in each of the two cases.

Finally, with reference to the shorter latency of the jaw-jerk as compared with that of the knee-jerk. I may observe that it does not militate against the view that both are phenomena of direct muscular excitation; the relative bulk of the muscles involved, and of the parts to be moved, in the two cases, explains the apparent discrepancy. The shortening of a small muscle, like the masseter, as judged by the movement of the jaw, must obviously consume less time than a contraction of such a mass as the quadriceps femoris in effecting the extension of a pendulous leg.

I shall conclude with a short recapitulation of what I, in common with others, hold to be the most likely hypothesis concerning the nature of tendon-reactions. In its normal condition, all muscular tissue remains in constant relation with the spinal grey matter, by means of afferent and efferent nervous fibres. Upon integrity of this nervous arc depends the so-called "tonicity" of muscle, as well as its property of contracting in response to the stimulus of sudden extension. All conditions that angment or impair tonicity modify like. wise this property. Hence the state of the tendon-reactions may be nsed as a test of the presence or absence of alterations in the muscular reflex spinal arc, without any direct appeal to its activity. 